\title{
Pavetta bridsoniae (Rubiaceae), a replacement name for a Zimbabwean species
}

\section{Paul I. Forster ${ }^{1}$}

Summary. The species name Pavetta muelleri Bridson is an illegitimate homonym of $P$. muelleri Bremek. A replacement name (P. bridsoniae P. I. Forst.) honouring Diane Bridson is proposed for this Zimbabwean species.

Key Words. Flora Zimbabwe, nomenclature, Pavetta muelleri.

\section{Introduction}

The genus Pavetta L. is widespread in the Old World tropics with between 350 and 400 species (Bridson 2003; Govaerts et al. 2011). Most species are found on the African continent, although outliers of the genus occur as far afield as Melanesia and Australia (Reynolds 1993; Govaerts et al. 2011).

During preparation of a Flora of Australia account for Pavetta, it was noticed that Pavetta muelleri Bridson (originally as 'mulleri') described from Zimbabwean material (Bridson 2001), was a later homonym for P. muelleri Bremek. from northern Australia (Reynolds 1993). A replacement name is proposed here, honouring Diane Bridson who worked for many years on Rubiaceae from Africa and Asia.

Pavetta bridsoniae P. I. Forst. nom. nov.

http:/ /www.ipni.org/urn:lsid:ipni.org:names:77121640-1
Pavetta muelleri Bridson in Kew Bull. 56: 586 (2001); Bridson, Fl. Zambesiaca 5(3): 573 (2003); non Bremekamp (1934: 124). Type: Zimbabwe, Nyanga Distr., Müller 2654 (holotype SRGH).

\section{References}

Bremekamp, C. E. B. (1934). A monograph of the genus Pavetta L. Repert. Spec. Nov. Regni Veg. 37: 1 - 208.

Bridson, D. M. (2001). Additional notes on Pavetta (Rubiaceae: Pavetteae) from Tropical Eastern and Southern Africa. Kew Bull. 56: 567 - 600.

(2003). Pavetta. In: G. V. Pope, Flora Zambesiaca Vol. 5 part 3. Royal Botanic Gardens, Kew for Flora Zambesiaca Managing Committee.

Govaerts, R., Ruhsam, M., Andersson, L., Robbrecht, E., Bridson, D. M., Davis, A. P., Schanzer, I. \& Sonké, B. (2011). World Checklist of Rubiaceae. Royal Botanic Gardens, Kew. Available at: http://www.kew.org/wcsp/rubiaceae

Reynolds, S. T. (1993). The genus Pavetta L. (Rubiaceae) in Australia. Austrobaileya 4: 21 - 49.

Accepted for publication 19 July 2012. Published online 8 September 2012

${ }^{1}$ Queensland Herbarium, Brisbane Botanic Gardens, Mt Coot-tha Road, Toowong, Queensland 4066, Australia. e-mail: paul.forster@derm.qld.gov.au 